$\int 46 \mathrm{Abs} 2$ öStGB) gemessen. Allenfalls bestehende regionale Unterschiede im Entlassungszeitpunkt innerhalb des letzten Strafdrittels wurden nicht untersucht.

27 Ein Sicherheitsnachteil ergibt sich nur insoweit, als sich das vom Rechtsbrecher ausgehende Risiko bei einer Strafrestaussetzung früher realisieren kann.

28 Siehe die Erläuterungen zur Regierungsvorlage, Nr 302 BlgNR XXIII. GP.

\section{Literatur}

Albrecht, H.J. (1982): Legalbewährung bei zu Geldstrafe und Freiheitsstrafe Verurteilten. Freiburg i. $\mathrm{Br}$.

Albrecht, H.J./Dünkel, F./Spiess, G. (1981): Empirische Sanktionsforschung und die Begründbarkeit von Kriminalpolitik. MschKrim, Jg. 64, S. $265-278$.

Aufsattler, W./Oswald, M./Geisler, W./Graßhoff, U. (1982): Eine Analyse richterlicher Entscheidungen über die Strafrestaussetzung nach $\$ 57 \mathrm{StGB}$. MschrKrim, Jg. 65, S. $305-317$.

Baumann, K.H./Maetze, W./Mey, H.G. (1983): Zur Rückfälligkeit nach Strafvollzug. MschrKrim, Jg. 66, S. 133 - 148.

Berckhauer, F./Hasenpusch, W. (1982): Rückfälligkeit entlassener Strafgefangener. Zusammenhänge zwischen Rückfall und Bildungsmaßnahmen im Vollzug. MschrKrim, Jg. 65, S. $318-334$.

Birklbauer, A./Hirtenlehner, H. (2007): Praxis und Wirksamkeit der bedingten Entlassung. Unveröffentlichter Forschungsbericht des Instituts für Strafrechtswissenschaften der Universität Linz.

Blossfeld, H.P./Rohwer, G. (2002): Techniques of Event History Modeling. Mahwah.

Böhm, A. (1996): Die spezialpräventiven Wirkungen der strafrechtlichen Sanktionen. In: Jehle, J.M. (Hrsg.): Kriminalprävention und Strafjustiz. Wiesbaden. S. $263-290$.
Bundesministerium für Inneres (verschiedene Jahrgänge): Sicherheitsbericht. Bericht der Bundesregierung über die Innere Sicherheit in Österreich. Wien.

Burgstaller, M./Császár, F. (1985a): Zur regionalen Strafenpraxis in Österreich. ÖJZ, Jg. 40, S. 1 -11, $43-47$.

Burgstaller, M./Császár, F. (1985b): Ergänzungsuntersuchungen zur regionalen Strafenpraxis. ÖJZ, Jg. 40, S. $417-427$.

Császár, F./Schäffner, M. (1991): Empirische Daten zur bedingen Entlassung nach dem StrÄG 1987. StPdG, Jg. 18, S. 143 - 175.

Diekmann, A. (1995): Empirische Sozialforschung Reinbek bei Hamburg.

Dünkel, F. (1980): Legalbewährung nach sozialtherapeutischer Behandlung. Eine empirische vergleichende Untersuchung. Berlin.

Dünkel, F. (2005): Entlassungsmodalitäten im Strafvollzug im europäischen Vergleich und Probleme der Kriminalprognose. In: Bundesministerium für Justiz (Hrsg.): Moderner Strafvollzug - Sicherheit und Resozialisierung. Wien: S. 37-69.

Eisenberg, U./Ohder, C. (1987): Aussetzung des Strafrestes zur Bewährung: Eine empirische Un tersuchung der Praxis am Beispiel von Berlin (West). Berlin.

Ellis, T./Marshall, P. (2000): Does Parole Work? A Post-Release Comparison of Reconviction Rates for Paroled and Non-Paroled Prisoners. The Australian and New Zealand Journal of Criminology, Jg. 33, S. $300-317$.

Hirtenlehner, H./Birklbauer, A. (2005): Rückfallsprävention durch Restaussetzung oder Austauschbarkeit der Entlassungsformen? Eine empirische Untersuchung am Beispiel von Sexualund Raubstraftätern in Österreich. NKP, Jg. 17, S $111-116$.

Hirtenlehner, H./Birklbauer, A. (2006): Lokale Gerichtskulturen - Die vernachlässigte Perspektive zur Erklärung regionaler Strafdisparitäten. Zugleich eine Bilanzierung des österreich- ischen Forschungsstandes zur regionalisierten Strafrechtspflege. JRP, Jg. 14, S. 287 - 298.

Hirtenlehner, H./Birklbauer, A./Wegscheider, H. (2002): Die bedingte Entlassung aus der Freiheitsstrafe. Eine empirische Analyse der vollzugsgerichtlichen Entscheidungsfindung bei Sexual- und Gewaltstraftätern. Wien.

Jehle, J.M. (2004): Die deutsche Rückfallstatistik Konzeption und Ertrag. In: Heinz, W./Jehle, J.M. (Hrsg.): Rückfallforschung. Wiesbaden. S. $145-$ 171.

Kensey, A. (2004): Conditional Release and the Prevention of Reoffending. In: Council of Europe (Hrsg.): Crime Policy in Europe: Good Practices and Promising Examples. Straßburg.

Klug, W. (2007): „Good Practice“ in der Arbeit mit Straftätern. SIO, Heft 3/07, S. 8 - 14

Kunz, K.L. (2004): Kriminologie. Bern.

Pilgram, A. (1974): Richterliche Kriterien und Erfolg der bedingten Entlassung von Strafgefangenen. Wien: unveröffentlichter Forschungsbericht des Instituts für Kriminalsoziologie.

Pilgram, A. (1991): Die erste österreichische Rückfallstatistik - ein Mittel zur Evaluation regionaler Strafenpolitik. ÖJZ, Jg. 46, S. 577 - 586.

Pilgram, A. (2004): Rückfallstatistische Untersuchungen in Österreich. In: Heinz, W./Jehle, J.M. (Hrsg.): Rückfallforschung. Wiesbaden. S. 319 334.

Pilgram, A. (2005): Die Praxis der (bedingten) Strafentlassung im regionalen Vergleich. Befunde auf neuer statistischer Grundlage. In: Bundesministerium für Justiz (Hrsg.): Moderner Strafvollzug Sicherheit und Resozialisierung. Wien: S. $79-$ 104

Pilgram, A./Hirtenlehner, H./Kuschej, H. (2000): Sozialer Ausschluss durch Kriminalisierung? Die Kriminalgerichtspraxis zwischen Strafen und Straffälligenhilfe. In: Pilgram, A./Steinert, H. (Hrsg.): Sozialer Ausschluss - Begriffe, Praktiken und Gegenwehr. Jahrbuch für Rechts- und Kriminalsoziologie 2000. Baden-Baden. S. 129 - 151.

\title{
Reform ist machbar
}

\section{Wolfgang Gratz / Arno Pilgram}

Die Insassenzahlen im österreichischen Strafvollzug lagen in den 1990er Jahren über mehr als 10 Jahre stabil um die 7.000. 2002 begannen sie rasch zu steigen, um sich ab 2005 bei 9.000 einzupendeln. Die Reaktion des Justizministers war die Forcierung von Fertigteilbauten auf dem Areal bestehender Anstalten, die Planung einer neuen Anstalt in Wien (wird derzeit weiter vorangetrieben) sowie der Versuch, in Rumänien eine von Österreich finanzierte Anstalt errichten zu lassen (das ist erfreulicherweise gescheitert). Die Überbelegung in den Justizanstalten und die Stagnation der Mitarbeiterzahlen in der Justizwache führte zum weiteren Vordringen des Verwahrvollzuges.

In dieser Situation fanden sich zu Beginn 2004 sieben ExpertInnen zur „Kriminalpolitischen Initiative“ (KI) mit dem Motto „Mehr Sicherheit durch weniger Haft“ zusammen. Im Frühsommer 2004 gingen sie mit Vorschlägen an die Öffentlichkeit, die Verminderung der Haftzahlen mit sicherheitswirksamen Strategien im Umgang mit Straftätern zu verbinden.

Den fünf Vorschlägen,

- den Tatbestand der Gewerbsmäßigkeit nur auf wirklich qualifizierte Delikte anzuwenden und Schöffengerichten zuzuweisen,

- die Verhängung der Untersuchungshaft an Gefährlichkeit und Verhältnismäßigkeit zu orientieren,

- für die Freiheitsstrafe mehr Gestaltungsspielraum vorzusehen (Elektronische Aufsicht und Gemeinnützige Leistungen als alternative Form des Strafvollzuges),

- die bedingte Entlassung und Nachbetreuung zu forcieren

- und die Qualität der Rechtsprechung durch Gerichtshilfe, Daten und Evaluation zu verbessern, wurde 2005 angesichts des steigenden Anteils von MigrantInnen unter den Inhaftierten ein sechster Vorschlag hinzugefügt:
- bei Strafgefangenen mit Aufenthaltsverbot von der Hälfte der Strafzeit vorläufig abzusehen, wenn sie an der Rückkehr in ihr Herkunftsland mitwirken.

Die KI verwies darauf, dass eine Realisierung ihrer Vorschläge auch ohne Überbelegung kriminalpolitisch angezeigt wäre und begründete dies entsprechend.

Es verwunderte kaum, dass unter der schwarzblauen Regierung die Vorschläge nicht umgesetzt wurden, obwohl sie öffentliche Aufmerksamkeit fanden (auch eine Enquete des Justizministeriums zur bedingten Entlassung ergab breite $\mathrm{Zu}$ stimmung zu den Vorschlägen).

Im Jänner 2007 wurde eine große Koalition mit einer sozialdemokratischen Bundesministerin für Justiz (Maria Berger) gebildet. Bereits Ende Februar 2007 präsentierte Ministerin Berger ein Haftentlassungspaket, dass weitestgehend die Vorschläge und auch das Motto der KI 
„Mehr Sicherheit durch weniger Haft“ übernahm. Zusätzlich strebte sie eine Ausweitung der Möglichkeit der Umwandlung von Freiheitsstrafen von bis zu neun (anstelle bisher sechs Monaten) in Geldstrafen sowie eine Amnestie 2008 anlässlich 90 Jahre Gründung der 1. Republik an.

Der Koalitionspartner reagierte darauf zunächst mit Kritik und weitgehender Ablehnung. Nach längeren Verhandlungen wurde Anfang Dezember im Nationalrat ein Reformgesetz beschlossen, das als Teilerfolg, - berücksichtigt man das schlechte Arbeitsklima der Koalition - sogar als ein erheblicher Fortschritt gewertet werden kann.

Von den sechs Vorschlägen der KI wurden drei aufgegriffen und in unterschiedlichem Maße umgesetzt:

A) Die alternative Vollziehung von Freiheitsstrafen wird nun zumindest insofern ermöglicht, als die Erbringung gemeinnütziger Leistungen anstelle der Ersatzfreiheitsstrafe für uneinbringliche Geldstrafen angeboten werden muss. Das Gesetz geht aber nicht so weit, Anstalten und Gefangenen die Option einzuräumen, kurze primäre Freiheitsstrafen durch Gemeinnützige Leistungen zu reduzieren.

B) Die bedingte Entlassung wird in mehrfacher Hinsicht gesetzlich gefördert.

- Es bedarf nun nicht mehr der (absoluten) Annahme, dass der bedingt zu Entlassene keine weiteren strafbaren Handlungen begehen werde, sondern der (relativen, also vergleichenden) Prognose, dass der Verurteilte durch die bedingte Entlassung nicht weniger als durch die weitere Verbüßung der Strafe von künftigen Straftaten abgehalten wird.

- Ein weiterer Vorteil der neuen Regelung ist das Abgehen von der starren zeitlichen Unterteilung in „Hälfte-Entlassungen“ und „Zwei-Drittel-Entlassungen" mit je unterschiedlichen Voraussetzungen. Nunmehr sind Strafgefangene nach Verbüßung der Hälfte der Freiheitsstrafe dann bedingt zu entlassen, sobald die Voraussetzungen vorliegen.

- Gemeinsam mit der künftig verstärkt anzuordnenden Bewährungshilfe und von Weisungen müsste deshalb die bedingte Entlassung wohl zum Regelfall werden, da kaum anzunehmen ist, dass eine voll verbüßte Haft (ohne weitere Betreuung und Kontrolle) stärker Rückfall vermeidend ist als eine bedingte Entlassung.

- Generalpräventive Überlegungen werden in den Hintergrund gedrängt, wenngleich erst nach zwei Dritteln der Strafe als Kriterium gänzlich gestrichen.

- Die bedingte Entlassung wird auch aus teilbedingten Strafen ermöglicht.

- Die Grundlagen für eine fachlich qualifizierte Entlassungsentscheidung werden durch Aufwertung der Begutachtungs- und Evaluationsstelle für Gewalt- und Sexualstraftäter verbessert.

Nicht erwärmen konnte sich der Gesetzgeber für die Übertragung der Entscheidung auf Senate mit einem Berufsrichter und zwei fachmännischen Laienrichtern, die mit Fragen der sozialen Reintegration beruflich vertrauter wären und denen dieses Kriterium wichtiger wäre als Strafzumessungsfragen.

C) Das „vorläufige Absehen vom Strafvollzug wegen Aufenthaltsverbotes“ wurde ebenfalls Gesetz, allerdings in einer stark abgeschwächten Variante. Von diesem Vollzugsverzicht werden leider einige Straftätergruppen ganz (Sexualstraftäter) oder teilweise ausgenommen (Körperverletzungstäter, wozu im Prinzip auch Drogenstraftaten zu zählen sind). Personen mit langen Strafen können davon gar nicht bzw. nicht bereits nach der Halbstrafe profitieren. Die Entscheidung obliegt dem Vollzugsgericht. Die restriktive Formulierung der Bestimmung und die praktischen Probleme bei der Zusammenarbeit zwischen Fremdenpolizeibehörde, Justizanstalten, Gerichten, NGOs für Rückkehrberatung und -hilfe werden dieser Bestimmung vermutlich einiges von ihrem Potenzial zur Haftentlastung nehmen.

Unberücksichtigt blieb von den Anregungen der Kriminalpolitischen Initiative die materiellrechtliche Korrektur an der Definition der Gewerbsmäßigkeit von Straftaten und an den zum Teil unverhältnismäßigen Strafsätzen in diesem Bereich. Von gesetzlichen Maßnahmen zur Reduzierung der U-Haft wurde ebenfalls Anstand genommen.

Als Erfolg der KI kann gewertet werden, dass in extensiver Auslegung des Strafvollzugsgesetzes im Jänner 2008 in zwei österreichischen Justizanstalten ein Modellprojekt startet, in dem Strafgefangene außerhalb der Anstalt wohnen und arbeiten, elektronisch überwacht und sozialarbeiterisch betreut werden.

Bleibt zu wünschen, dass für die Implementation des Haftentlastungspakets auch die notwendigen Ressourcen im Vollzug (Entlassungsvorbereitung, Begutachtungsstelle für Gewalt- und Sexualstraftäter, Rückkehrberatung für Gefangene aus Drittstaaten) und bei Neustart (Bewährungshilfekapazität) zur Verfügung stehen. Im Sinne der erforderlichen Qualitätssicherung in der Strafrechtspflege ist weiters eine wissenschaftliche Evaluation des Haftentlastungspakets zu wünschen.

Die Erfahrungen mit der weitgehend verpufften Reform der bedingten Entlassung 1987 legen es aber nahe, die Umsetzung der nunmehrigen Novelle gut zu begleiten, beispielsweise eine generelle Anhebung der Dauer von Haftstrafen vor dem Hintergrund der regelmäßigen bedingten Entlassung zu verhindern. Als Maßnahmen der Überprüfung und Qualitätssicherung sind Statistiken über Entlassungspraktiken der einzelnen Vollzugsgerichte und über Rückfälle der Entlassenen angezeigt. Diese Daten sollten Grundlage eines entwicklungsorientierten Dialogs aller Beteiligten (Richter, Staatsanwälte, Sachverständige, Strafvollzug, Nachbetreuung und -behandlung) sein, der auf regionaler und nationaler Ebene Fragen auf empirischer und nachvollziehbarer Basis zu beantworten sucht: Welche Ergebnisse und Erfolge haben wir? Wie liegen wir im Vergleich zu den anderen? Welche Schlüsse ziehen wir daraus?

Eine mögliche künftige Aufgabe der KI kann darin bestehen, ein wirksam öffentlich kommuniziertes Monitoring der Umsetzung zu betreiben. Die verschiedenen erforderlichen Kompromisse werden möglicherweise relativ bald den Ruf nach einer Reform der Reform erforderlich machen.

Wenngleich die Initiative der KI wahrscheinlich aus der spezifisch österreichischen Situation zu erklären ist (Die Kleinheit des Landes erleichtert wirkungsvolles Auftreten), lohnt es sich vielleicht doch, ihre Erfolgsfaktoren darzulegen:

- Klare Fokussierung auf Reformen, die unmittel- oder mittelbar auf Reduzierung der Haft abzielen 
- Starke internationale Orientierung: der Großteil der Vorschläge beruht auf einer Anpassung erprobter ausländischer Modelle an die österreichische Situation

- In der Zusammensetzung der KI: Balance von Gemeinsamkeit (kriminalpolitisches Grundverständnis) und Verschiedenheit in den professionellen Zugängen (Recht, Sozialwissenschaft, Sozialarbeit)

- Das Zusammenspiel der verschiedenen Kapitalien der KI-Mitglieder (theoretisches und praktisches Wissen, Feldkompetenz, Reputation, Beziehungen zu Multiplikatoren und Entscheidungsträgern) ergibt eine potente Gruppe.
- Zivilcourage: Wenngleich kein KI-Mitglied ein höheres berufliches Risiko einging, war einigen ein „Naserümpfen“ oder „hochgezogene Augenbrauen“ von KollegInnen und Vorgesetzten sicher.

- Kontinuität auf Sparflamme: Es wurde ein ökonomischer und arbeitsteiliger Arbeitsmodus gefunden, der die einzelnen Mitglieder relativ wenig belastete.

- Kommunikation der Vorschläge auf verschiedenen Kanälen: Pflege vorhandener Kontakte zu JournalistInnen in Verbindung mit drei Pressekonferenzen und einer Presseaussendung, informelle Kontakte zu Justizpolitikern und Beamten des Justizministeriums, Vorsprachen bei drei verschiedenen MinisterIn- nen, Referate auf Tagungen und Publikationen in Fachzeitschriften.

- Parteipolitische Äquidistanz: Alle Vorschlägen beruhen auf kriminalpolitischen Überlegungen, die zwar eine größere oder weniger große Nähe zu den Vorstellungen einzelner politischer Parteien haben, nicht aber auf parteipolitischen Positionen.

Trotz aller Bemühungen sind Initiativen wie die KI von der politischen Großwetterlage abhängig. Es kann sich aber lohnen, bei ungünstigen klimatischen Bedingungen gegensteuernd aufzutreten. Bei politischen Veränderungen sind dann rasche Teilerfolge möglich.

\section{Hamburger Strafvollzug - Wege und Irrwege Zentrale Fakten einer verfehlten Strafvollzugspolitik}

\section{Fünf Hauptsünden der hamburgischen Strafvollzugspolitik seit 2001}

1. Geld verschleudert: Ausbau der

JVA Billwerder um weitere ca. 400, auf rund 800 Plätze

Mit Bürgerschaftsdrucksache vom 7.5. 2002 (Drs. 17/802) beantragten Kusch/ Lüdemann 30.260.000 € für die Erweiterung der JVA Billwerder um 400 Plätze des geschlossenen Vollzuges (Billwerder II). Dieses Geld wurde in den Sand gesetzt. Zusätzlich wird Hamburg mit jährlichen Betriebsmittelkosten von ca. 1.400.000€ belastet. Wie konnte es dazu kommen? Drei Antworten:

Falsche Prognose: Kusch/Lüdemann erwarteten von 2002 bis 2004 einen Anstieg der Zahl der Gefangenen um $17 \%$ auf 3.600. Daran hielten er, seine Behörde, der Senat und die Fraktionen der Koalition fest, obwohl ihnen und der Öffentlichkeit alsbald und wiederholt der Unsinn (und die Geschichtsblindheit!) dieser Prognose vorgehalten worden war. Schon im Jahr der Mitteleinwerbung (2002) wurden nicht die vorausgesagten 3.266 (Drs. 17/802, S. 3), sondern nur 3.085 Gefangene gezählt. Bereits im Januar 2004 sank sie unter die Grenze von 3.000. Nach kontinuierlichem Rückgang seither lag sie im Durchschnitt des Jahres 2006 bei $2.549,2007$ bei 2.227 Gefangenen.

Überflüssige Haftplätze: Für diese ca. 2.250 Gefangenen sind, nach Abschluss laufender Umbauten, 3.202 Haftplätze vorhanden. „Jede vierte Gefängniszelle“, so „Die Welt“ am 6. 12. 2006, „steht leer". Es war unsinnig, Billwerder II zu bauen - insbesondere, wenn die mehr als 100 Haftplätze im Übergangsvollzug und in der Sozialtherapie erhalten geblieben wären (siehe dazu Punkt 5).

Blockierte Kontrollen: Der Vorgang rührt an Grundfragen eines sachgerechten Verwaltungshandelns. Die erste Rate für Billwerder II in Höhe von 8,8 Mio € war 2004 fällig. Genug Zeit, sollte man meinen, um die Planung anhand der Fakten zu überprüfen, zumal die Bauaufträge erst am 30. 1. 2004 erteilt wurden (s. Broschüre der Justizbehörde „So viel ist sicher" vom Februar 2006, S. 22). Aber alle Warnungen wurden missachtet. Wichtige Hamburger Medien versagten; Senat und Bürgerschaftsmehrheit winkten das Projekt durch. Die sonst äußerst strengen und fachlich versierten Kontrollen in der Finanzbehörde und im Planungsstab der Senatskanzlei blieben wirkungslos. Zu fragen ist, ob und ggf. von wem die fachbehördlichen und behördenübergreifenden Sicherungen außer Kraft gesetzt worden sind.

Was sagt die Justizbehörde heute? In einem Interview mit „Die Welt“ vom 5. 1. 2007 beklagt Senator Lüdemann die „Geschichtsvergessenheit der Opposition“. Auch Rot-Grün habe 350 Haftplätze neu errichten wollen. Jedoch gibt es zwischen den Planungen der Jahre 1999/2000 und denen von Kusch/Lüdemann der Jahre 2001/2002 drei große Unterschiede: Die Belegungsfähigkeit des geschlossenen Vollzuges sollte nach damaligen Plänen nur um 175 Plätze angehoben, weitere 175 sollten dagegen zum Abbau von Saalbelegungen genutzt werden. Angenommen wurde ein Zuwachs der Gefangenenzahlen von 2000 bis 2004 von jährlich nur einem Prozent. Selbst dies wurde, wie es Brauch war, von der Finanzbehörde und dem Planungsstab energisch hinterfragt. Das führte dazu, dass der Bau nicht vor 2004 beginnen sollte, und dass die Realisierung zwingend an den jährlichen Nachweis der Belegungszahlen gebunden wurde. Diese Sperre gegenüber fachbehördlichen Durchmarschund Geldverschwendungsgelüsten hätte das Projekt vermutlich gestoppt.

2. Umbau um jeden Preis: die offene Anstalt Billwerder wird geschlossener Vollzug

Kusch/Lüdemann haben alsbald nach Amtsübernahme angeordnet, die vom Vorgängersenat als offene Anstalt mit 380 Plätzen konzipierte Einrichtung Billwerder I in geschlossenen Vollzug umzuwandeln. Dafür wurden 14.000.000€ veranschlagt, bewilligt und vergeudet. Die Umrüstung war baufachlich überflüssig, weil auch 